\title{
The Simulation of Trace Gas Pollution Adsorption on Silica Gel
}

\author{
A Rong \\ School of Aviation Science and Engineering \\ Beijing University of Aeronautics and Astronautics \\ Beijing, China \\ ivory_118@126.com \\ Pang Liping \\ School of Aviation Science and Engineering \\ Beijing University of Aeronautics and Astronautics \\ Beijing, China \\ pangliping@buaa.edu.cn
}

\author{
Liu Meng \\ School of Aviation Science and Engineering \\ Beijing University of Aeronautics and Astronautics \\ Beijing, China \\ Liumeng@buaa.edu.cn \\ Yu Qingni \\ National key laboratory of human factors, China \\ Astronaut Research and Training Center \\ Beijing, China \\ yuqingni@139.com
}

\author{
Wang Jun \\ School of Aviation Science and Engineering \\ Beijing University of Aeronautics and Astronautics \\ Beijing, China \\ wangjun@buaa.edu.cn
}

\begin{abstract}
Silica gel has a potential ability to adsorb water vapor and contaminant together, so it has been focused recently in indoor air environment. In this paper, the performance of silica gel adsorbing trace gas pollutions in isothermal column is studied through theoretical methods. The model of the adsorption column system was set up based on mass conservation, linear driving force model and Henry's Law. An implicit differential method was used to solve the model in the Matlab environment and to obtain the breakthrough curves. Seven simulation experiments were conducted to discuss the effect of overall mass transfer coefficient, Henry parameter and gas velocity on adsorption process. From the result it indicated that the overall mass transfer coefficient impacts the breakthrough point enormously. Henry parameter reflects the adsorption capacity of adsorbent particle, high Henry constant affords long life-span of adsorbent. Furthermore, high efficient and long-term use of adsorbent cannot obtain without reasonable gas velocity.
\end{abstract}

Keywords-silica gel; adsorption; gas pollution; simulation; breakthrough curve;

\section{INTRODUCTION}

Silica gel is a form of silicon dioxide, which is amorphous and highly porous polar sorbent. In 1919, Walter A. Patrick ${ }^{[1]}$, a chemistry professor of Johns Hopkins University, patented the synthetic route for producing silica gel possessing great adsorbing power, which promoted the applications of silica gel. Soon, silica gel was found to adsorb $0.4 \mathrm{~kg}$ of moisture per $\mathrm{kg}$ of gel by Dehler (1940) ${ }^{[2]}$, employed widely as dehumidifying material in the food industries, electronic industries, medical products, as well as in solar desiccant cooling systems.
Although silica gel, the amorphous and highly porous adsorbent, is used in a wide range of applications, the adsorption capacity of trace gas pollutants has yet to be dug, only a modicum of research are published in the public journals. Fang et al. (2008) $)^{[3]}$ designed two experiments use four pure chemicals (formaldehyde, ethanol, toluene and 1,2-dichloroethane) to investigate the effect of a desiccant wheel in the ventilation system. Their results indicate that the desiccant wheel could remove the trace gas pollutants with an average efficiency of $94 \%$ or higher. Zhang et al. (2007) ${ }^{[4]}$ conducted a theoretical study of simultaneous water and Volatile Organic Compounds (VOCs) adsorption and desorption in a silica gel desiccant rotor. Hines and Ghosh $(1993)^{[5]}$ studied the competitive adsorption of propane-propylene mixture on silica gel, which revealed that silica gel has the greatest selectivity for propylene compared with molecular sieve $13 \mathrm{X}$ and activated carbon. Ghosh and Hines $(1990)^{[6]}$ obtained the adsorption equilibrium experiment data of acetaldehyde, propionaldehyde and butyraldehyde on silica gel respectively. Strøm-Tejsen et al. (2008) ${ }^{[7]}$ proved that the improvement of the indoor air quality has a certain effect when the silica gel wheel was used by investigating passengers' perception. All the study showed that the silica gel has an adsorption capability to remove both trace gases pollutants and water.

In this paper, we will analyze the performance of silica gel to absorb trace gas pollutants by theory analysis. In the theoretical study, the trace gas pollutants adsorbed on silica gel column is modeled based on mass conservation, linear driving force model and Henry's Law. Seven simulation experiments are conducted to discuss the effect of overall mass transfer coefficient, Henry parameter and gas velocity during adsorption process. 


\section{MATHEMATICAL MODEL}

The system described here is an isothermal adsorption column as shown in Fig .1, which was $270 \mathrm{~mm}$ long with a $5.3 \mathrm{~mm}$ inner radius packed with porous adsorbent. Finepore silica gel (product of Qingdao Haiyang Chemical Co., Ltd.) employed as the adsorbent in this study has a specific surface area of $750 \mathrm{~m}^{2} / \mathrm{g}$, a total pore volume of $0.40 \mathrm{~cm}^{3} / \mathrm{g}$, and the average pore size of $2.5 \mathrm{~nm}$. The packing density was $755.45 \mathrm{~kg} / \mathrm{m}^{3}$, and the void fraction was 0.252 .

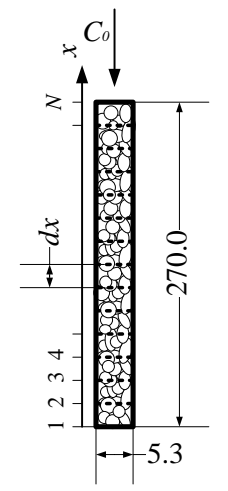

Figure 1. Adsorption column packed with silica gel.

A single adsorbate passed downwardly through the adsorption column with carrier gas (Nitrogen) by constant flow rate. The three major simplifying assumptions were made in the following analysis:

- The inlet pressure, temperature, gas velocity and concentration are assumed to be constant.

- No chemical reactions occur in the adsorption column.

- The gas stream through the adsorption column is treated as plug flow.

With the above assumptions, the flow in the adsorption column can be regarded as one dimensional axial flow. However, for the perpendicular plug flow, if the boundary layer is thin, compared to the length of the adsorption column, the axial dispersion can be neglected. Kramers et al. $(1953)^{[8]}$, Klinkenberg et al. (1953) $)^{[9]}$ and de Carvalho et al. (2003) ${ }^{[10]}$ proofed that a conservative criterion for this assumption to be valid is $L / d p>20$. In this study, $L / d p=135$. So the influence of axial dispersion can be neglected. The mass balance on the adsorption column, considered axial dispersion, can be described by the following equation:

$$
u \frac{\partial C}{\partial x}+\frac{\partial C}{\partial t}+\frac{1-\varepsilon_{b}}{\varepsilon_{b}} \rho_{b} \frac{\partial \bar{q}}{\partial t}=0
$$

where $x$ is the axial coordinate, $\mathrm{m} ; t$ is the time coordinate, s; $C$ is the concentration of adsorbate in bulk flow, $\mu \mathrm{g} / \mathrm{m}^{3} ; \bar{q}$ is the average amount of adsorbate adsorbed per unit mass of adsorbent, $\mu \mathrm{g} / \mathrm{kg} ; u$ is the gas velocity in the column, $\mathrm{m} / \mathrm{s} ; \varepsilon_{b}$ is the void fraction of the column; $\rho_{b}$ is the packing density of the column, $\mathrm{kg} / \mathrm{m}^{3}$.

The mass transfer rate equation was established by the Linear Driving Force (LDF) model, which is frequently and successfully used for analysis adsorptive process in adsorption column e.g. Sircar et al. (2000) ${ }^{[11]}$ and Amari et al. $(2010)^{[12]}$.The mass transfer rate equation can be written as

$$
\frac{\partial \bar{q}}{\partial t}=k\left(q^{*}-\bar{q}\right)
$$

where $k$ is overall mass transfer coefficient, $\mathrm{s}^{-1} ; q^{*}$ is equilibrium concentration of the adsorbate adsorbed per unit mass of adsorbent, $\mu \mathrm{g} / \mathrm{kg}$.

In the low-pressure limit, the equilibrium relative to the gas-solid interface can be described by the Henry's law, so the adsorption is linear in pressure and the isotherm has a slope equal to the equilibrium constant. In this study, the partial pressure of the adsorbate is low enough to the Henry's law. So the equilibrium equation can be written as

$$
q^{*}=K C
$$

where $K$ is Henry parameter, called Henry's adsorption constant also, $\mathrm{m}^{3} / \mathrm{kg}$.

So (2) becomes

$$
\frac{\partial \bar{q}}{\partial t}=k(K C-\bar{q})
$$

Above all, the adsorption column system can be modeled by (1) and (4) partial differential equations.

With initial condition

$$
C=C_{e n v} \text { for } z>0, t=0
$$

And boundary conditions

$$
\begin{aligned}
& C=C_{0} \text { for } z=0, t \geq 0 \\
& \frac{\partial C}{\partial x}=0 \text { for } z=L, t \geq 0
\end{aligned}
$$

where $C_{e n v}$ is an initial adsorbate concentration in the adsorption column, $\mu \mathrm{g} / \mathrm{m}^{3} ; \quad C_{0}$ is inlet adsorbate concentration in the fluid, $\mu \mathrm{g} / \mathrm{m}^{3}$.

An implicit difference method, which uses the backward difference at both time and space domain, was used to solve the mathematical model. Although this method needs a complex algorithm to solve, its numerical solutions are always stability. The entire adsorption column is divided into $N-1$ equal domains as showed in Fig .1. The entire time domain is divided into $I-1$ same domains. Define a variable $X$ at time $i$ and position $n$ as $X_{n}^{i}$. Equation (1) and (4) can be final rewritten as

$$
\left\{\begin{array}{l}
-u \frac{1}{\Delta x} C_{n-1}^{i}+\left(u \frac{1}{\Delta x}+\frac{1}{\Delta t}\right) C_{n}^{i}+\frac{1-\varepsilon_{b}}{\varepsilon_{b}} \rho_{b} \frac{1}{\Delta t} \overline{q_{n}^{i}}=\frac{1}{\Delta t} C_{n}^{i-1}+\frac{1-\varepsilon_{b}}{\varepsilon_{b}} \rho_{b} \frac{1}{\Delta t} \overline{q_{n}^{i-1}} \\
-k K C_{n}^{i}+\left(k+\frac{1}{\Delta t}\right) \overline{q_{n}^{i}}=\frac{1}{\Delta t} \overline{q_{n}^{i-1}}
\end{array}\right.
$$

where $i$ is time node, $i=2,3, . ., I ; n$ is position node, $n=2,3, \ldots, N$.

The silica gel must be dried before packing, and the adsorbate need to be conditioned for at least 2 hours under carrier gas flow after filled in the adsorption column. Hence the initial and boundary conditions become

$$
C_{n}^{1}=0, \quad n=2,3, \cdots N \text { and } C_{1}^{1}=C_{0}
$$

\section{DISCUSSION}

The model of adsorption column described by (8) and (9) was numerical solved in Matlab environment. Several simulation experiments were conducted to discuss the effect of overall mass transfer coefficient, Henry parameter 
and gas velocity on adsorption process. These 7 simulation cases summarized in TABLE I will be classified four conditions to discuss:

- Condition 1 (Case 1, 2, 3, and 4): Henry parameter and gas velocity are constant, the overall mass transfer coefficient is the only variable.

- Condition 2 (Case 1, and 5): When the overall mass transfer coefficient is on a high value, only Henry parameter changes.

- Condition 3 (Case 4, and 6): When the overall mass transfer coefficient is on a low value, only Henry parameter changes.

- Condition 4 (Case 3, and 7): Henry parameter and the overall mass transfer coefficient are constant, and gas velocity is the only variable.

TABLE I. SimULATION CASES

\begin{tabular}{|c|c|c|c|}
\hline $\begin{array}{c}\text { Simulation } \\
\text { cases }\end{array}$ & $\begin{array}{c}\text { overall mass } \\
\text { transfer coefficient } \\
\left(\mathbf{s}^{-1}\right)\end{array}$ & $\begin{array}{c}\text { Henry } \\
\text { parameter } \\
\left(\mathbf{m}^{\mathbf{3}} / \mathbf{k g}\right)\end{array}$ & $\begin{array}{c}\text { gas } \\
\text { velocity } \\
(\mathbf{m} / \mathbf{s})\end{array}$ \\
\hline 1 & 0.9 & 0.65 & 0.05 \\
\hline 2 & 0.09 & 0.65 & 0.05 \\
\hline 3 & 0.009 & 0.65 & 0,05 \\
\hline 4 & 0.0009 & 0.65 & 0.05 \\
\hline 5 & 0.9 & 1.30 & 0.05 \\
\hline 6 & 0.0009 & 1.30 & 0.05 \\
\hline 7 & 0.009 & 0.65 & 0.1 \\
\hline
\end{tabular}

The simulation results of adsorption process are described by the breakthrough curves, as shown in Fig .2, 3,4 , and 5 . The breakthrough curves are represented the evolution of the ratio of $C / C_{0}$ as a function of time, where $\mathrm{C}$ is the concentration of adsorbate in the gas stream at the outlet of adsorption column, and $C_{0}$ is the concentration of adsorbate in the gas stream at the inlet.

\section{A. Effect of Overall Mass Transfer Coefficient}

The overall mass transfer coefficient is the only variable in condition 1 . By simulating the case $1,2,3$, and 4 respectively, four breakthrough curves are acquired, shown in Fig .2.

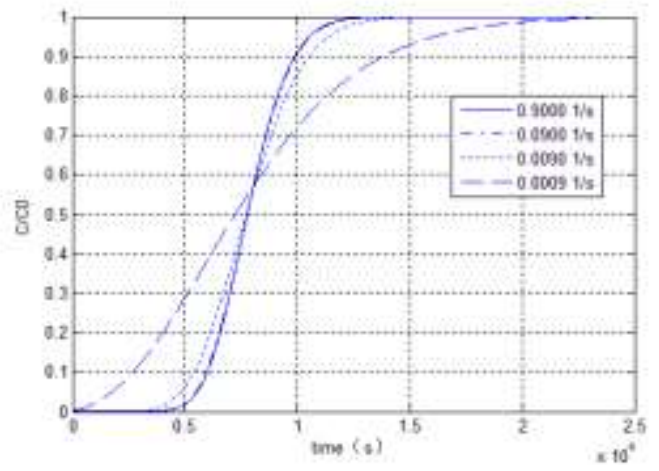

Figure 2. Breakthrough curves of simulation Case 1, 2, 3, and 4.

From Fig .2, we can observe the influences of the overall mass transfer coefficient on the adsorption process.

With the geometric growth of the overall mass transfer coefficient, the breakpoint occurred later, the depth of the adsorption zone became shorter, and the slope of the breakthrough curve got the steep. These phenomena reveal that the adsorbent showed increasable adsorption capacity and efficient use with the growth of the overall mass transfer coefficient.

The simulated curves of Case 1 and Case 2 are almost overlapping, though their overall mass transfer coefficients are $0.9 \mathrm{~s}^{-1}$ and $0.09 \mathrm{~s}^{-1}$, respectively. Therefore, when the value of overall mass transfer coefficient is high enough, its influence will be weakened in the adsorption process.

When the value of overall mass transfer coefficient is too low as in Case 4, the breakpoint rapidly occurred almost overlap with zero moment. Hence the column adsorption capacity decreased obviously when the overall mass transfer coefficient is relatively low.

\section{B. Effect of Henry Parameter}

From the results of simulation Case 1 and Case 2, we find that the effect of the overall mass transfer coefficient on the adsorption capacity can be ignored when it increases to be over a certain extent. Thus, in order to investigate the effect of Henry parameter with different overall mass transfer coefficient, two conditions are considered in this section.

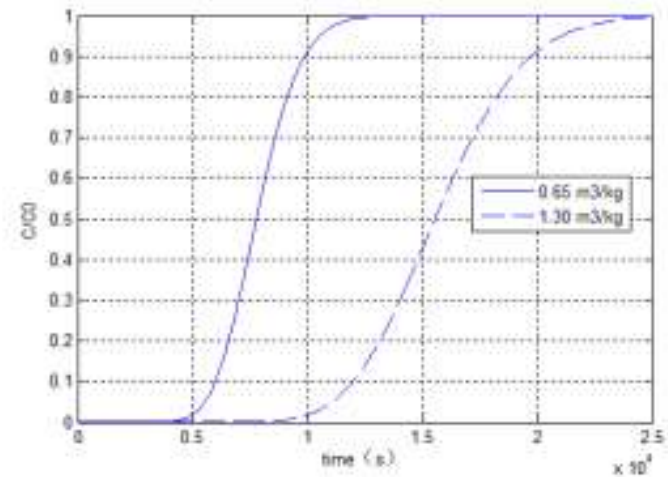

Figure 3. Breakthrough curves of simulation case 1, and 5

Simulation Condition 2 (Case 1 and 5), as shown in Fig .3, concerns a relatively high overall mass transfer coefficient of $0.9 \mathrm{~s}^{-1}$. It's obviously showed in Fig .3 that the increase of the Henry parameter affects the adsorption capacity greatly. Furthermore, when the overall mass transfer coefficient increases to be a certain extent, which means the adsorbate can pass through the boundary layer easily and diffuse adequately, the Henry parameter will become the main factor for an adsorption process.

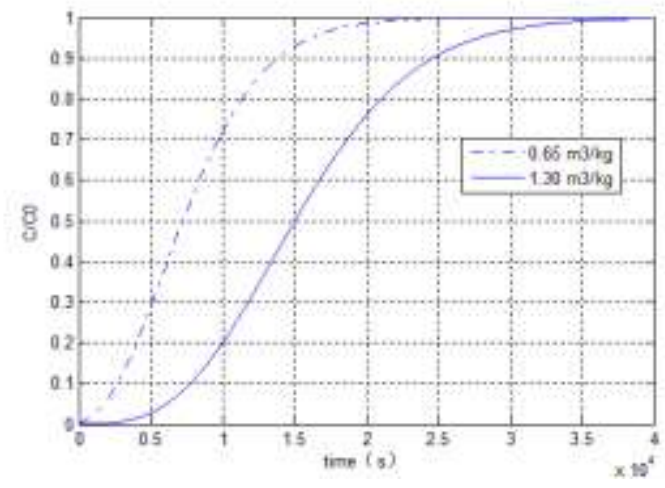

Figure 4. Breakthrough curves of simulation case 4 , and 6 . 
Simulation Condition 3 (Cases 4 and 6), as shown in Fig .4, was conducted to discuss the effect of Henry parameter on a relatively low overall mass transfer coefficient of $0.0009 \mathrm{~s}^{-1}$. The simulation results are In these cases, the breakpoint curves almost exactly overlaps although the Henry parameter increased from $0.65 \mathrm{~m}^{3} / \mathrm{kg}$ to $1.30 \mathrm{~m}^{3} / \mathrm{kg}$, and only the saturation time increases in the adsorption process. As the outlet adsorbate concentration is concerned, we find when the overall mass transfer coefficient is relatively low, the external film mass transfer resistance and the solid diffusion resistance are high, and the Henry parameter's increase does not improve the breakthrough point.

\section{Effect of Gas Velocity}

Condition 4 concerns the other one of the mainly factor during the adsorption process. Case 3 and 7 simulated the adsorption process with a gas velocity of $0.05 \mathrm{~m} / \mathrm{s}$ and $0.10 \mathrm{~m} / \mathrm{s}$, respectively.

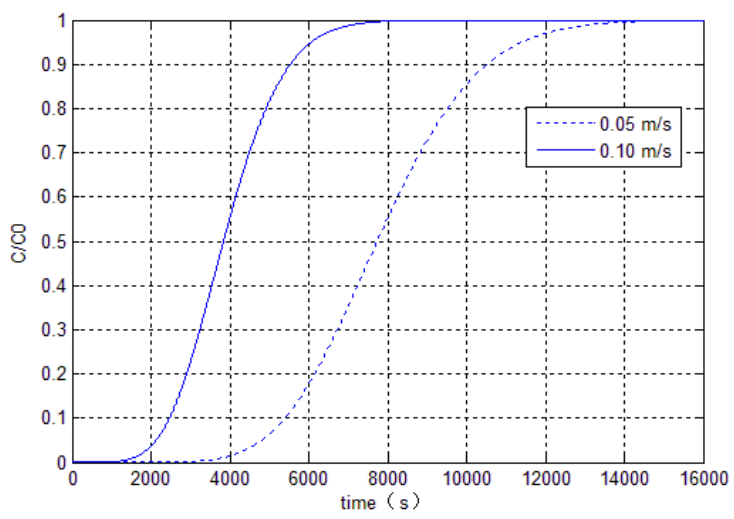

Figure 5. Breakthrough curves of simulation case 3, and 7.

In simulation Case 3, and 7, as shown in Fig .5, we discuss the influence of the gas velocity on the situation that both the overall mass transfer coefficient and the Henry parameter are constant by decreasing the gas velocity, the breakthrough time and exhaustion time increases. This shows that the slow gas velocity will contribute a great interaction between the adsorbate and adsorbent. Therefore, the low gas velocity can lead to an effectively increase of the column adsorption capacity and a long time to approach saturation.

\section{CONCLUSIONS}

In this paper, we investigated the absorption kinetics of the single contaminant passed downwardly through the silica gel adsorption column. A mathematical model for column adsorption processes was developed to simulate and analyze the adsorption process. According to the above simulation analysis, we obtain the effects of three parameters on the adsorption process, and draw the following conclusions:

- The overall mass transfer coefficient which can represent the adsorbate's ability to pass through the boundary layer and to diffuse in the pore of adsorbent, impacts the breakthrough point enormously. But with the increase of the overall mass transfer coefficient, the adsorbate sufficient diffused but the adsorption capacity limited by the Henry parameter.

- The Henry parameter can indicate the adsorbent's ability to adsorb the adsorbate onto the porous surface of silica gel, high Henry constant affords long life-span of adsorbent

- The gas velocity can determine the contact time between the gas stream and adsorbent. The adverse impact of a relatively fast gas velocity is enormous. High efficient and long-term use of adsorbent cannot obtain without reasonable gas velocity.

However, more work has to be done to study the silica gel's ability absorb water vapor and contaminant together, and to make the model more appropriate.

\section{ACKNOWLEDGMENT}

This study was sponsored by the Open Funding Project of National Key Laboratory of Human Factors Engineering project number SYFD140051811K.

\section{REFERENCES}

[1] W. A. Patrick, Silica Gel and Process of Making Same. U.S. Patent and Trademark Office, Washington, DC. Dec. 1919, No. 1,297,724.

[2] F. C. Dehler, "Silica gel adsorption." Chem Metall Eng, vol. 37, 1940, pp. 307-310.

[3] L. Fang, G. Zhang, and A. Wisthaler. "Desiccant wheels as gas phase absorption (GPA) air cleaners: evaluation by PTR - MS and sensory assessment." Indoor Air, vol. 18, 2008, pp: 375-385, doi:10.1111/j.1600-0668.2008.00538.x.

[4] G. Zhang, Y. F. Zhang, and L. Fang. "Theoretical study of simultaneous water and VOCs adsorption and desorption in a silica gel rotor." Indoor Air, vol. 18, 2008, pp: 37-43, doi:10.1111/j.1600-0668.2007.00502.x.

[5] T. K. Ghosh, H. Lin, and A. L. Hines. "Hybrid adsorptiondistillation process for separating propane and propylene." Industrial \& engineering chemistry research, vol. 32, 1993, pp 2390-2399.

[6] T. K. Ghosh, and A. L. Hines. "Adsorption of acetaldehyde, propionaldehyde, and butyraldehyde on silica gel." Separation science and technology, vol. 25, 1990, pp: 1101-1115.

[7] P. Strøm - Tejsen, D. Zukowska, L. Fang, D. R. Space, and D. P.Wyon. "Advantages for passengers and cabin crew of operating a gas - phase adsorption air purifier in 11 - $\mathrm{h}$ simulated flights." Indoor air, vol. 18, 2008, pp:172-181, doi:10.1111/j.16000668.2007.00511.x.

[8] H. Kramers, and G. Alberda. "Frequency response analysis of continuous flow systems." Chemical Engineering Science, vol, 1953, pp: 173-181.

[9] A. Klinkenberg, H. J. Krajenbrink, and H. A. Lauwerier. "Diffusion in a fluid moving at uniform velocity in a tube." Industrial \& Engineering Chemistry, vol. 45, 1953, pp:1202-1208.

[10] de Carvalho, J. R. F., and J. M. P. Q. Delgado. "Effect of fluid properties on dispersion in flow through packed beds." AIChE journal, vol. 49, 2003, pp:1980-1985.

[11] S. Sircar, and J. R. Hufton. "Why does the linear driving force model for adsorption kinetics work?" Adsorption, vol. 6, 2000, pp: 137-147.

[12] A. Amari, M. Chlendi, A. Gannouni, and A. Bellagi. "Experimental and theoretical studies of VOC adsorption on acidactivated bentonite in a fixed-bed adsorber." Industrial \& Engineering Chemistry Research, vol. 49, 2010, pp: 11587-11593, doi:10.1021/ie100296n. 